\title{
Selecting Parameters for the Rainbow Signature Scheme - Extended Version -
}

\author{
Albrecht Petzoldt ${ }^{1}$, Stanislav Bulygin ${ }^{2}$, and Johannes Buchmann ${ }^{1,2}$ \\ 1 Technische Universität Darmstadt, Department of Computer Science \\ Hochschulstraße 10, 64289 Darmstadt, Germany \\ \{apetzoldt, buchmann\}@cdc.informatik.tu-darmstadt.de \\ 2 Center for Advanced Security Research Darmstadt - CASED \\ Mornewegstraße 32, 64293 Darmstadt, Germany \\ \{johannes.buchmann, Stanislav.Bulygin\}@cased.de
}

\begin{abstract}
Multivariate public key cryptography is one of the main approaches to guarantee the security of communication in a post-quantum world. One of the most promising candidates in this area is the Rainbow signature scheme, which was first proposed by J. Ding and D. Schmidt in 2005. In this paper we develop a model of security for the Rainbow signature scheme. We use this model to find parameters for Rainbow over GF(16), GF(31) and GF(256) which, under certain assumptions, guarantee the security of the scheme for now and the near future.
\end{abstract}

Keywords: Multivariate cryptography, Rainbow signature scheme, parameters

\section{Introduction}

To guarantee the security of communication it is important to have fast and secure signature schemes. One major field of application for them is the authenticity of data and information, for example software updates.

One of the most promising candidates in this area is the Rainbow signature scheme, which was presented by J. Ding and D. Schmidt in [DS05]. Similarly to other multivariate schemes like $3 i C^{-} p$ [DW07] and Projected Flash [PC01], [DY07] it is very efficient and provides fast signature generation and verification. In opposite to classical schemes, e.g. RSA or ECDSA, Rainbow is believed to be secure against attacks with quantum computers [BB08].

In the last years a lot of work has been done to study the security of multivariate schemes and many attacks were proposed. Among these are direct attacks on which a lot of work was done [YC07], [Fa99] as well as rank attacks which were introduced in [CS94] by Coppersmith and Stern to attack the Birational Permutation Scheme and later improved by a number of other researchers [YC05], [BG06]. A good overview of these attacks can be found in [GC00]. Special attacks on Rainbow-like schemes were proposed by Ding and Yang in [DY08]. There have also been some attempts to derive appropriate parameters from the complexities of these attacks [CC08]. However, it is still an open problem how we have to adapt the parameters of multivariate schemes to future developments in cryptanalysis and computing power.

In this paper we try to answer this question for the Rainbow signature scheme. We start with the security model of Lenstra and Verheul [LV00] to compute necessary security levels for the years 2010 to 2050. After that we look at the known attacks against the Rainbow signature scheme. Here, we concentrate mainly on two attacks, namely the direct attack and the Rainbow-BandSeparation attack. To study the complexity of these two attacks, we carried out a large number of own experiments, for which we used MAGMA [BC06], which contains an efficient implementation 
of Faugeres $F_{4}$ [Fa99] algorithm for computing Gröbner bases. We use the results of these experiments to find appropriate parameters for Rainbow over the underlying fields $G F(16), G F(31)$ and $G F(256)$. Finally, we compare Rainbow schemes over the different fields in terms of key sizes and signature lengths. One of our main results here is, that we get the smallest keys for Rainbow schemes over $G F(31)$, whereas we get the shortest signatures when using Rainbow over $G F(16)$.

The structure of the paper is as follows: In Section 2 we describe the Rainbow signature scheme. Section 3 describes our model of security for the Rainbow scheme. In Section 4 we take a closer look at the complexities of the direct and the Rainbow-Band-Separation attack and give concrete parameter sets for Rainbow over the fields $G F(16), G F(31)$ and $G F(256)$. Section 5 summarizes our results and compares the Rainbow schemes over the different ground fields in terms of key sizes and signature length. Finally, Section 6 concludes the paper.

\section{Multivariate Public Key Cryptography}

Multivariate Public Key Cryptography is one of the main approaches for secure communication in a post-quantum world. The principle idea is to choose a multivariate system $F$ of quadratic polynomials which can be easily inverted (central map). After that one chooses two affine linear invertible maps $S$ and $T$ to hide the structure of the central map. The public key of the cryptosystem is the composed map $P=S \circ F \circ T$ which is difficult to invert. The private key consists of $S$, $F$ and $T$ and therefore allows to invert $P$.

There are several ways to build the central map $F$. One approach are the so called BigFieldSchemes like Matsumoto-Imai [MI88] and HFE [Pa96] with many variations and improvements [BB08], [Di04], [PC01]. On the other hand, we have the so called SingleField family with schemes like UOV [KP99] and Rainbow [DS05]. Recently, a third family called MediumField has been proposed which contains schemes like $\ell$-iC [DW07].

\subsection{The principle of Oil and Vinegar (OV)}

One way to create easily invertible multivariate quadratic systems is the principle of Oil and Vinegar, which was first proposed by J. Patarin in [Pa97].

Let $K$ be a finite field (e.g. $K=G F\left(2^{8}\right)$ ). Let $o$ and $v$ be two integers and set $n=o+v$. Patarin suggested to choose $o=v$. After this original scheme was broken by Kipnis and Shamir in [KS98], it was recommended in [KP99] to choose $v>o$ (Unbalanced Oil and Vinegar (UOV)). In this Section we describe the more general approach UOV.

We set $V=\{1, \ldots, v\}$ and $O=\{v+1, \ldots, n\}$. Of the $n$ variables $x_{1}, \ldots, x_{n}$ we call $x_{1}, \ldots, x_{v}$ the Vinegar variables and $x_{v+1}, \ldots, x_{n}$ Oil variables. We define $o$ quadratic polynomials $f_{k}(\mathbf{x})=f_{k}\left(x_{1}, \ldots, x_{n}\right)$ by

$$
f_{k}(\mathbf{x})=\sum_{i \in V, j \in O} \alpha_{i j}^{(k)} x_{i} x_{j}+\sum_{i, j \in V, i \leq j} \beta_{i j}^{(k)} x_{i} x_{j}+\sum_{i \in V \cup O} \gamma_{i}^{(k)} x_{i}+\eta^{(k)}(k \in O)
$$

Note that Oil and Vinegar variables are not fully mixed, just like oil and vinegar in a salad dressing.

The map $F=\left(f_{v+1}(\mathbf{x}), \ldots, f_{n}(\mathbf{x})\right)$ can be easily inverted. First, we choose the values of the $v$ Vinegar variables $x_{1}, \ldots, x_{v}$ at random. Such we get a system of $o$ linear equations in the $o$ variables $x_{v+1}, \ldots, x_{n}$ which can be solved by Gaussian Elimination. (If the system doesn't have a solution, choose other values of $x_{1}, \ldots, x_{v}$ and try again). 


\subsection{The Rainbow Signature Scheme}

In [DS05] J. Ding and D. Schmidt proposed a new signature scheme called Rainbow, which is based on the idea of Oil and Vinegar.

Let $K$ be a finite field (e.g. $\left.K=G F\left(2^{8}\right)\right)$ and $S$ be the set $\{1, \ldots, n\}$. Let $v_{1}, \ldots, v_{u+1}, u \geq 1$ be integers such that $0<v_{1}<v_{2}<\cdots<v_{u}<v_{u+1}=n$ and define the sets of integers $S_{i}=\left\{1, \ldots, v_{i}\right\}$ for $i=1, \ldots, u$. We set $o_{i}=v_{i+1}-v_{i}$ and $O_{i}=\left\{v_{i}+1, \ldots, v_{i+1}\right\}(i=1, \ldots, u)$. The number of elements in $S_{i}$ is $v_{i}$ and we have $\left|O_{i}\right|=o_{i}$. For $k=v_{1}+1, \ldots, n$ we define multivariate quadratic polynomials in the $n$ variables $x_{1}, \ldots, x_{n}$ by

$$
f_{k}(\mathbf{x})=\sum_{i \in O_{l}, j \in S_{l}} \alpha_{i, j}^{(k)} x_{i} x_{j}+\sum_{i, j \in S_{l}, i \leq j} \beta_{i, j}^{(k)} x_{i} x_{j}+\sum_{i \in S_{l} \cup O_{l}} \gamma_{i}^{(k)} x_{i}+\eta^{(k)},
$$

where $l$ is the only integer such that $k \in O_{l}$. Note that these are Oil and Vinegar polynomials with $x_{i}, i \in S_{l}$ being the Vinegar variables and $x_{j}, j \in O_{l}$ being the Oil variables.

The map $F(\mathbf{x})=\left(f_{v_{1}+1}(\mathbf{x}), \ldots, f_{n}(\mathbf{x})\right)$ can be inverted as follows: First, we choose $x_{1}, \ldots, x_{v_{1}}$ at random. Hence we get a system of $o_{1}$ linear equations (given by the polynomials $f_{k}\left(k \in O_{1}\right)$ ) in the $o_{1}$ unknowns $x_{v_{1}+1}, \ldots, x_{v_{2}}$, which can be solved by Gaussian Elimination. The so computed values of $x_{i}\left(i \in O_{1}\right)$ are put into the polynomials $f_{k}(\mathbf{x})\left(k>v_{2}\right)$ and a system of $o_{2}$ linear equations (given by the polynomials $\left.f_{k}\left(k \in O_{2}\right)\right)$ in the $o_{2}$ unknowns $x_{i}\left(i \in O_{2}\right)$ is obtained. By repeating this process we can get values for all the variables $x_{i}(i=1, \ldots, n)^{3}$.

The Rainbow signature scheme is defined as follows:

Key Generation The private key consists of two invertible affine maps $L_{1}: K^{m} \rightarrow K^{m}$ and $L_{2}: K^{n} \rightarrow K^{n}$ and the map $F=\left(f_{v_{1}+1}(\mathbf{x}), \ldots, f_{n}(\mathbf{x})\right)$. Here, $m=n-v_{1}$ is the number of components of $F$.

The public key consists of the field $K$ and the composed map $P(\mathbf{x})=L_{1} \circ F \circ L_{2}(\mathbf{x}): K^{n} \rightarrow K^{m}$.

Signature Generation To sign a document $d$, we use a hash function $\mathbf{h}: K^{*} \rightarrow K^{m}$ to compute the value $\mathbf{h}=\mathbf{h}(d) \in K^{m}$. Then we compute recursively $\mathbf{x}=L_{1}^{-1}(\mathbf{h}), \mathbf{y}=F^{-1}(\mathbf{x})$ and $\mathbf{z}=L_{2}^{-1}(\mathbf{y})$. The signature of the document is $\mathbf{z} \in K^{n}$. Here, $F^{-1}(\mathbf{x})$ means finding one (of the possibly many) pre-image of $\mathbf{x}$.

Verification To verify the authenticity of a signature, one simply computes $\mathbf{h}^{\prime}=P(\mathbf{z})$ and the hashvalue $\mathbf{h}=\mathbf{h}(d)$ of the document. If $\mathbf{h}^{\prime}=\mathbf{h}$ holds, the signature is accepted, otherwise rejected.

The size of the public key is (for $K=G F\left(2^{8}\right)$ )

$$
\text { size }(\text { public key })=m \cdot\left(\frac{n \cdot(n+1)}{2}+n+1\right)=m \cdot \frac{(n+1) \cdot(n+2)}{2} \text { bytes, }
$$

the size of the private key

$$
\text { size }(\text { private key })=m \cdot(m+1)+n \cdot(n+1)+\sum_{l=1}^{u} o_{l} \cdot\left(v_{l} \cdot o_{l}+\frac{v_{l} \cdot\left(v_{l}+1\right)}{2}+v_{l+1}+1\right) \text { bytes. }
$$

The length of the needed hash value is $m$ bytes, the length of the signature is $n$ bytes. The scheme is denoted by Rainbow $\left(v_{1}, o_{1}, \ldots, o_{u}\right)$. For $u=1$ we get the original UOV scheme.

\footnotetext{
${ }^{3}$ It may happen, that one of the linear systems does not have a solution. If so, one has to choose other values of $x_{1}, \ldots x_{v_{1}}$ and try again.
} 


\section{Our Model of Security}

In this Section we describe the model underlying our parameter choices below. We base on the approach of Lenstra and Verheul [LV00].

\subsection{The model}

In [LV00] Lenstra and Verheul developed a security model, which they used to find appropriate parameters for symmetric cryptography and some asymmetric schemes. The main points of their model are:

1. Security margin: a definition of the term "adequate security".

2. Computing environment: the expected change in computational resources available to attackers.

3. Cryptanalytic development: the expected development in cryptanalysis.

In the following we take a closer look at these items.

Security margin To decide, whether a given scheme offers adequate security, one has to define the term "adequate security". [LV00] defines it by the security offered by DES in 1982. That is, in 1982 a computational effort of $5 \cdot 10^{5}$ MIPS years provided an adequate security. We follow this definition.

Computing environment Here [LV00] use a slightly modified version of Moore's law, which states that the amount of computing power and random access memory one gets for 1 dollar doubles every $t$ months. Our default setting of $t$ is 18, see [LV00]

Another thing we have to take into account, is the budget of an attacker, which might increase over time. The variable $b>0$ is defined as the number of years it takes on average for an expected two-fold increase of a budget. Statistical data says, that the US Gross National product (in today's prices) doubles about every ten years. So our default setting for $b$ is 10 .

Cryptanalytic Development The number $r>0$ is defined to be the number of months it is expected to take on average for cryptanalytic developments affecting Multivariate Public Key Cryptosystems to become twice as effective.

Under the assumption, that the pace of cryptanalytic findings in the area of multivariate cryptography will not vary dramatically from those in the field of classical cryptosystems, our default setting for $r$ is $r=18$.

After having developed concrete security levels based on these three items, Lenstra and Verheul analyzed known attacks against several schemes to get concrete parameter sets.

Analogous to [LV00], we will use "Infeasible number of MIPS years" (IMY) to define security requirements for the Rainbow signature scheme. Given that breaking DES takes $5 \cdot 10^{5}$ MIPS years, which was infeasible to do in year 1982, we get the number of MIPS years that are infeasible to break in the year $y$ by the formula

$$
I M Y(y)=5 \cdot 10^{5} \cdot 2^{12(y-1982) / t} \cdot 2^{(y-1982) / b} \quad \text { MIPS years. }
$$

With our default settings we get

$$
I M Y(y)=2^{\frac{23}{30} \cdot y-1500.6} \text { MIPS years }
$$

So far, we have not considered the possible advances in cryptanalysis. To cover these, we have to adapt the upper formula slightly. So, a cryptosystem, which shall be secure in the year $y$, must reach the security level

$$
\text { Security level }(y) \geq I M Y(y) \cdot 2^{12(y-2009) / r} \text { MIPS years } \stackrel{r=18}{=} 2^{\frac{43}{30} \cdot y-2839.9} \text { MIPS years }
$$


To translate this security bound into the corresponding number of field multiplications, we use a data-point computed by J. Ding et al. in [DY08]. There the authors solve a system of 37 quadratic equations in 22 variables over $G F\left(2^{8}\right)$ in about $1.06 \cdot 10^{6}$ seconds on a single $2.2 \mathrm{GHz}$ Opteron machine by XL-Wiedemann. This corresponds to approximately 329.7 MIPS years ${ }^{4}$. Since the complexity of the system is about $2^{46.7} \mathrm{~m}$, we get

$$
1 \text { MIPS year }=3.49 \cdot 10^{11} \mathrm{~m}
$$

Such we get

$$
\text { Security } \operatorname{level}(y) \geq 2^{\frac{43}{30} \cdot y-2801.5} \mathrm{~m}
$$

For our experiments (see next section) we use a single core Opteron $2.7 \mathrm{GHz} \mathrm{CPU}$ with 128 GB RAM. Since this CPU achieves about 10200 MIPS, we get

$$
\text { Security } \operatorname{level}(y) \geq 2^{\frac{43}{30} \cdot y-2853.2} \mathrm{~s}
$$

\subsection{Security level of Rainbow}

In this subsection we look at the known attacks against the Rainbow signature scheme. We will find, that the security of the scheme is mainly given by the complexities of two attacks, namely the direct and the Rainbow-Band-Separation attack and therefore can be said to be the minimum of those two complexities.

The known attacks against the Rainbow Signature Scheme are:

1. direct attacks [BB08], [Ya07]: Direct attacks use equation solvers like XL and its derivatives as well as Gröbner Basis algorithms: Buchberger, $F_{4}$, and $F_{5}$. The complexity is approximately given as

$$
C_{\text {direct }}(q, m, n)=C_{M Q(q, m, n)},
$$

where $C_{M Q(q, m, n)}$ denotes the complexity of solving a "generic" system of $m$ quadratic equations in $n$ variables over a field with $q$ elements.

2. Rainbow-Band-Separation attack [DY08]

$$
C_{\mathrm{RBS}}(q, m, n)=C_{M Q(q, m+n-1, n)}
$$

3. MinRank attack [GC00], [YC05]

$$
C_{\mathrm{MR}}\left(q, m, n, v_{1}\right)=\left[q^{v_{1}+1} \cdot m \cdot\left(n^{2} / 2-m^{2} / 6\right)\right] \mathrm{m}
$$

4. HighRank attack [GC00], [DY08]

$$
C_{\mathrm{HR}}\left(q, n, o_{u}\right)=\left[q^{o_{u}} \cdot n^{3} / 6\right] \mathrm{m}
$$

5. UOV attack [KP99]

$$
C_{\mathrm{UOV}}\left(q, n, o_{u}\right)=\left[q^{n-2 \cdot o_{u}-1} \cdot o_{u}^{4}\right] \mathrm{m}
$$

6. UOV-Reconciliation attack [BB08], [DY08]

$$
C_{\mathrm{UOVR}}\left(q, m, n, o_{u}\right)=C_{M Q\left(q, m, n-o_{u}\right)}
$$

7. Attacks against the hashfunction

Here, $\mathrm{m}$ stands for the number of field multiplications needed during the attack.

\footnotetext{
${ }^{4}$ The given processor achieves about 9800 MIPS (SiSoft Sandra)
} 
Defending a Rainbow scheme against the attacks from the items 3 to 7 is relatively easy:

Proposition 1: A Rainbow instance over $G F(q)$ with parameters $v_{1}, o_{1}, \ldots, o_{u}$ (see Section 2.2) , for which the items

1. $v_{1} \geq \frac{\ell}{\lg _{2}(q)}-1$

2. $o_{u} \geq \frac{\ell}{\lg _{2}(q)}$

3. $n-2 \cdot o_{u} \geq \frac{\ell}{\lg _{2}(q)}+1$

hold, has a security level of $\ell$ bits against the MinRank, the HighRank and the UOV attack.

Proof.

$$
\begin{gathered}
C_{\mathrm{MR}}\left(q, m, n, v_{1}\right)=\left[q^{v_{1}+1} \cdot m \cdot\left(n^{2} / 2-m^{2} / 6\right)\right] \mathrm{m} \stackrel{1 \cdot}{\geq}\left[2^{a \cdot \ell / a} \cdot m \cdot\left(n^{2} / 2-m^{2} / 6\right)\right] \mathrm{m}>2^{\ell} \mathrm{m} \\
C_{\mathrm{HR}}\left(q, n, o_{u}\right)=\left[q^{o_{u}} n^{3} / 6\right] \mathrm{m} \stackrel{2 .}{\geq}\left[2^{a \cdot \ell / a} \cdot n^{3} / 6\right] \mathrm{m}>2^{\ell} \mathrm{m} \\
C_{\mathrm{UOV}}\left(q, n, o_{u}\right)=\left[q^{n-2 o_{u}-1} \cdot o_{u}^{4}\right] \mathrm{m} \stackrel{3}{\geq}\left[2^{a \cdot \ell / a} \cdot o_{u}^{4}\right] \mathrm{m}>2^{\ell} \mathrm{m} \quad \square
\end{gathered}
$$

Together, the complexities of the HighRank- and the UOV-attack give us a lower bound for the number of variables we need in a secure Rainbow Scheme. Namely, we get

$$
n \geq \frac{3 \cdot \ell}{\lg _{2}(q)}+1
$$

To defend the scheme against the UOV-Reconciliation attack, we need $v_{1} \geq o_{u}$. Then, the algebraic part of the attack leads to an underdetermined system of quadratic equations which is as difficult to solve as a direct attack against the original scheme.

In order to prevent attacks on the hashfunction, one has to choose the number $m$ of equations in the system large enough such that a birthday attack against a hashfunction with $\lg _{2}\left(q^{m}\right)$ bit is infeasible.

In opposite to this, how one has to choose the parameters of Rainbow in order to defend the scheme against the direct and the Rainbow-Band-Separation attack, it not quite as clear and depends closely on the cardinality of the underlying field.

In the next section, we will take a closer look at these two complexities for the underlying fields $G F(16), G F(31)$ and $G F(256)$ and try to find appropriate parameter sets for Rainbow over these fields.

\section{Parameter choice}

In this section we want to find appropriate parameter sets for the Rainbow Signature Scheme over the underlying fields $G F(16), G F(31)$ and $G F(256)$.

The number of equations we need in our Rainbow Scheme is mainly determined by

- The complexity of a direct attack and

- Attacks against the hashfunction

Then number of variables in the scheme is mainly determined by

- The complexity of the RBS-attack

- The complexity of the UOV-attack and HighRank attack

In the following three subsections we look at Rainbow Schemes over $G F(16), G F(31)$ and $G F(256)$. 


\subsection{Rainbow Schemes over GF(16)}

Rank- and UOV attacks Table 1 gives the parameter restrictions set by Rank and UOV attacks. To prevent attacks with the UOV-Reconciliation attack, one should also have $v_{1} \geq o_{u}$.

\begin{tabular}{|l|c|c|c|c|}
\hline years & $\begin{array}{c}\text { MinRank } \\
v_{1} \geq\end{array}$ & $\begin{array}{c}\text { HighRank } \\
o_{u} \geq\end{array}$ & $\begin{array}{c}\text { UOV-Attack } \\
n-2 o_{u} \geq\end{array}$ & $\begin{array}{c}\text { HR+UOV } \\
n \geq\end{array}$ \\
\hline 2010 & 19 & 20 & 21 & 61 \\
$2011-2013$ & 20 & 21 & 22 & 64 \\
$2014-2015$ & 21 & 22 & 23 & 67 \\
$2016-2018$ & 22 & 23 & 24 & 70 \\
$2019-2021$ & 23 & 24 & 25 & 73 \\
$2022-2024$ & 24 & 25 & 26 & 76 \\
$2025-2027$ & 25 & 26 & 27 & 79 \\
$2028-2029$ & 26 & 27 & 28 & 82 \\
$2030-2032$ & 27 & 28 & 29 & 85 \\
$2033-2035$ & 28 & 29 & 30 & 88 \\
$2036-2038$ & 29 & 30 & 31 & 91 \\
$2039-2041$ & 30 & 31 & 32 & 94 \\
$2042-2043$ & 31 & 32 & 33 & 97 \\
$2044-2046$ & 32 & 33 & 34 & 100 \\
$2047-2049$ & 33 & 34 & 35 & 103 \\
$2050-2052$ & 34 & 35 & 36 & 106 \\
\hline
\end{tabular}

Table 1. Parameter restrictions for Rainbow over GF(16) according to Proposition 1

Direct attacks We carried out a large number of experiments of solving Rainbow systems over $G F(16)$ with MAGMA's $F_{4}$ algorithm. Before we could apply the MAGMA function GroebnerBasis, we had to convert the underdetermined Rainbow systems into determined ones by guessing at some of the variables. Since an underdetermined system with $m$ equations in $n>m$ variables has approximately $16^{(n-m)}$ solutions, it can be expected that our determined systems have a solution. By guessing at additional variables we created overdetermined systems to see whether this reduces the time needed to compute a Gröbner Basis. When doing so, one has to run the algorithm several times to find a solution of the original system.

Figure 1 shows the results of these experiments. As the figure shows, for more than 35 equations we get the best results by guessing at ten additional variables. The time MAGMA needs to solve $16^{10}$ of these overdetermined systems can be estimated as

$$
R T_{F_{4}}(16, m)=2^{1.67 \cdot m+3.4} s(m \geq 35)
$$

The number of equations we need to reach our security level, is therefore given as

$$
m \geq \frac{\log _{2}(\operatorname{Security} \operatorname{level}(y))-3.4}{1.67}
$$

Note that the numbers $m$ we get by this formula would lead to hash lengths which are not secure. So the number of equations in our schemes is determined by the hash length.

RBS-attack Due to the complexity of the UOV-attack we get an impression how many variables we need in our Rainbow scheme (see Table 1). To see whether this number is big enough to defend the scheme against the Rainbow-Band-Separation (RBS) attack, we carried out some experiments to estimate the running time of this attack. In the first step of the RBS attack one has to solve 


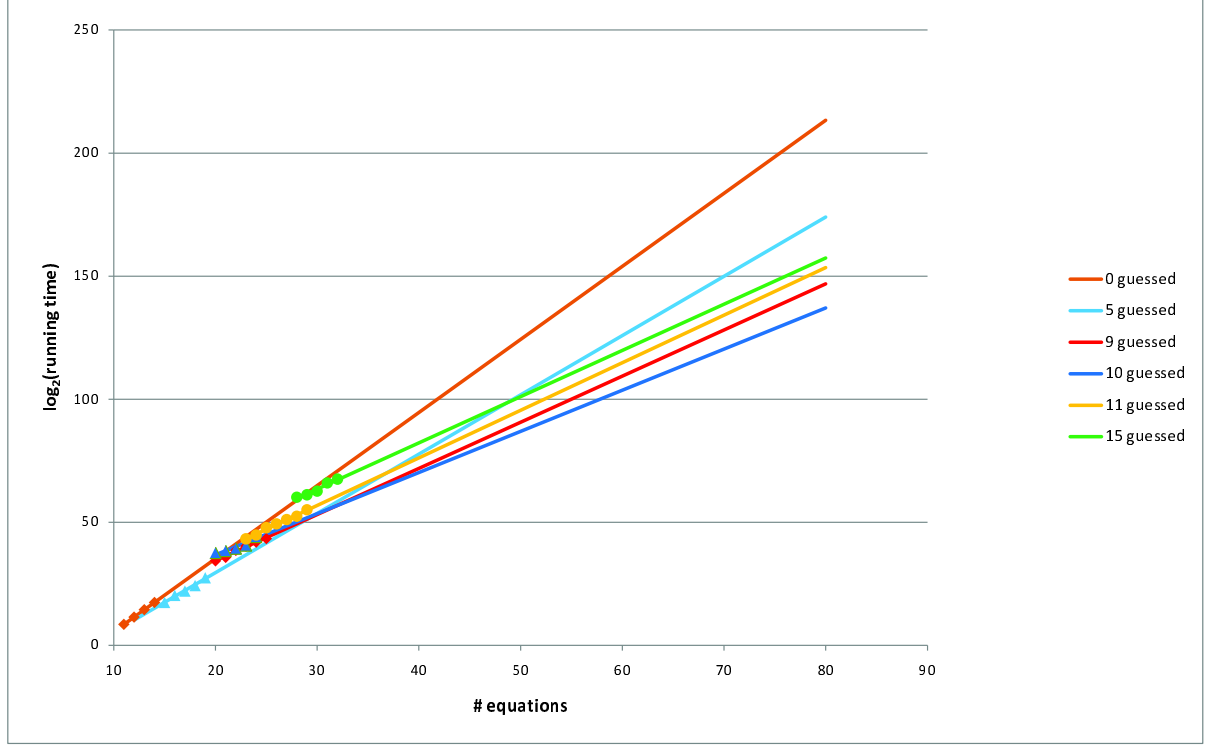

Fig. 1. Running time of the direct attack against Rainbow schemes over GF(16) with guessing

an overdetermined system of $m^{\prime}=m+n-1$ equations in $n$ variables. The running time of the RBS attack is mainly given by the time needed to solve this system.

For different values of $m$ and $n$ we carried out experiments to find the time MAGMA needs to solve this initial system. Table 2 shows the results.

As figure 2 shows, for a Rainbow scheme over GF(16) with $m$ equations and $n=\frac{3}{2} \cdot(m-1)$ variables the running time of the RBS attack is as least as high as the running time of the direct attack (dotted line in the figure). Therefore, the values of $n$ shown in table 1 are high enough. Table 3 shows the proposed parameters for Rainbow Schemes over GF(16).

\subsection{Rainbow Schemes over GF(31)}

In [CC09] Chen et al. suggested to define multivariate schemes over the field GF(31). Using this field seems to be especially appropriate on PC's with modern CPU's supporting the SSE vector instruction set extensions. In this Section we want to find the optimal parameters for the Rainbow Signature Scheme over GF(31).

Table 4 gives the parameter restrictions set by Rank and UOV attacks. To prevent attacks with the UOV-Reconciliation attack, one should also have $v_{1} \geq o_{u}$.

Direct attacks We carried out some experiments of solving Rainbow systems over $G F(31)$ with MAGMA's $F_{4}$ algorithm. Again, we had to convert the underdetermined Rainbow systems into determined ones by guessing at some of the variables, before we could apply the MAGMA function GroebnerBasis. Since an underdetermined system with $m$ equations in $n$ variables has approximately $31^{(n-m)}$ solutions, it can be expected that our determined systems have a solution. By 


\begin{tabular}{|c||c|c|c|c|c|}
\hline \multirow{4}{*}{$n=2 \cdot(m-1)$} & $\mathrm{m}$ & 8 & 9 & 10 & 11 \\
\cline { 2 - 6 } & $\mathrm{n}$ & 14 & 16 & 18 & 20 \\
\cline { 2 - 6 } & & $35.2 \mathrm{~s}$ & $798 \mathrm{~s}$ & $9527 \mathrm{~s}$ & $161738 \mathrm{~s}$ \\
& & $28 \mathrm{MB}$ & $209 \mathrm{MB}$ & $753 \mathrm{MB}$ & $2763 \mathrm{MB}$ \\
\hline \hline \multirow{5}{*}{$n=\frac{5}{3} \cdot(m-1)$} & $\mathrm{m}$ & 7 & 10 & 13 & \\
\cline { 2 - 6 } & $\mathrm{n}$ & 10 & 15 & 20 & \\
\cline { 2 - 6 } & & $0.15 \mathrm{~s}$ & $53.2 \mathrm{~s}$ & $30127 \mathrm{~s}$ & \\
\hline \hline \multirow{5}{*}{$n=\frac{3}{2} \cdot(m-1)$} & & $6.6 \mathrm{MB}$ & $35 \mathrm{MB}$ & $2032 \mathrm{MB}$ & \\
\cline { 2 - 6 } & $\mathrm{m}$ & 9 & 11 & 13 & 15 \\
\cline { 2 - 6 } & $\mathrm{n}$ & 12 & 15 & 18 & 21 \\
\cline { 2 - 6 } & & $0.8 \mathrm{~s}$ & $49.8 \mathrm{~s}$ & $1172 \mathrm{~s}$ & $72298 \mathrm{~s}$ \\
& & $8.5 \mathrm{MB}$ & $32.2 \mathrm{MB}$ & $170.0 \mathrm{MB}$ & $2916 \mathrm{MB}$ \\
\hline
\end{tabular}

Table 2. Running time of the RBS attack against Rainbow Schemes over GF(16)

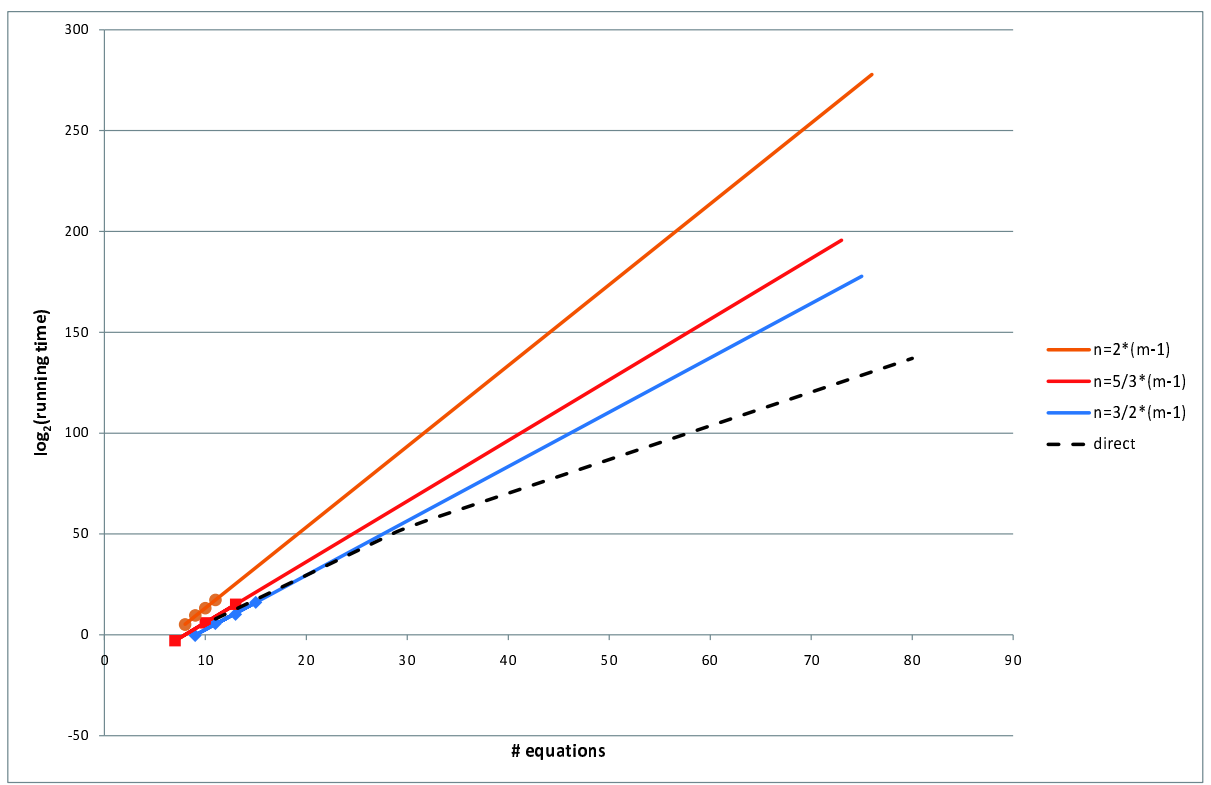

Fig. 2. Running time of the RBS attack against Rainbow over GF(16) for different ratios of $m$ and $n$ 


\begin{tabular}{|c|c|c|c|c|c|c|c|}
\hline years & $\begin{array}{c}\text { hash } \\
\text { size (bit) }\end{array}$ & $(\mathrm{m}, \mathrm{n})$ & $\begin{array}{l}\text { public key } \\
\text { size }(\mathrm{kB})\end{array}$ & $\begin{array}{c}\text { example scheme } \\
\left(v_{1}, o_{1}, o_{2}\right)\end{array}$ & $\begin{array}{c}\text { private key } \\
\text { size }(\mathrm{kB})\end{array}$ & $\begin{array}{l}\text { signature } \\
\text { size (bit) }\end{array}$ & IMY \\
\hline 1982 & & & & & & & $5.00 \cdot 10^{5}$ \\
\hline 2010 & 160 & $(40,61)$ & 38.1 & $(21,20,20)$ & 26.4 & 244 & $1.45 \cdot 10^{12}$ \\
\hline 2011 & 168 & $(42,64)$ & 44.0 & $(22,21,21)$ & 30.3 & 256 & $2.47 \cdot 10^{12}$ \\
\hline 2012 & 168 & $(42,64)$ & 44.0 & $(22,21,21)$ & 30.3 & 256 & $4.19 \cdot 10^{12}$ \\
\hline 2013 & 168 & $(42,64)$ & 44.0 & $(22,21,21)$ & 30.3 & 256 & $7.14 \cdot 10^{12}$ \\
\hline 2014 & 176 & $(44,67)$ & 50.4 & $(23,22,22)$ & 34.6 & 268 & $1.21 \cdot 10^{13}$ \\
\hline 2015 & 176 & $(44,67)$ & 50.4 & $(23,22,22)$ & 34.6 & 268 & $2.07 \cdot 10^{13}$ \\
\hline 2016 & 184 & $(46,70)$ & 57.4 & $(24,23,23)$ & 39.2 & 280 & $3.52 \cdot 10^{13}$ \\
\hline 2017 & 184 & $(46,70)$ & 57.4 & $(24,23,23)$ & 39.2 & 280 & $5.98 \cdot 10^{13}$ \\
\hline 2018 & 184 & $(46,70)$ & 57.4 & $(24,23,23)$ & 39.2 & 280 & $1.02 \cdot 10^{14}$ \\
\hline 2019 & 192 & $(48,73)$ & 65.0 & $(25,24,24)$ & 44.2 & 292 & $1.73 \cdot 10^{14}$ \\
\hline 2020 & 192 & $(48,73)$ & 65.0 & $(25,24,24)$ & 44.2 & 292 & $2.94 \cdot 10^{14}$ \\
\hline 2021 & 192 & $(48,73)$ & 65.0 & $(25,24,24)$ & 44.2 & 292 & $5.01 \cdot 10^{14}$ \\
\hline 2022 & 200 & $(50,76)$ & 73.3 & $(26,25,25)$ & 49.6 & 304 & $8.52 \cdot 10^{14}$ \\
\hline 2023 & 200 & $(50,76)$ & 73.3 & $(26,25,25)$ & 49.6 & 304 & $1.45 \cdot 10^{15}$ \\
\hline 2024 & 200 & $(50,76)$ & 73.3 & $(26,25,25)$ & 49.6 & 304 & $2.47 \cdot 10^{15}$ \\
\hline 2025 & 208 & $(52,79)$ & 82.3 & $(27,26,26)$ & 55.5 & 316 & $4.20 \cdot 10^{15}$ \\
\hline 2026 & 208 & $(52,79)$ & 82.3 & $(27,26,26)$ & 55.5 & 316 & $7.14 \cdot 10^{15}$ \\
\hline 2027 & 208 & $(52,79)$ & 82.3 & $(27,26,26)$ & 55.5 & 316 & $1.21 \cdot 10^{16}$ \\
\hline 2028 & 216 & $(54,82)$ & 91.9 & $(28,27,27)$ & 61.8 & 328 & $2.07 \cdot 10^{16}$ \\
\hline 2029 & 216 & $(54,82)$ & 91.9 & $(28,27,27)$ & 61.8 & 328 & $3.52 \cdot 10^{16}$ \\
\hline 2030 & 224 & $(56,85)$ & 102.3 & $(29,28,28)$ & 68.6 & 340 & $5.98 \cdot 10^{16}$ \\
\hline 2031 & 224 & $(56,85)$ & 102.3 & $(29,28,28)$ & 68.6 & 340 & $1.02 \cdot 10^{17}$ \\
\hline 2032 & 224 & $(56,85)$ & 102.3 & $(29,28,28)$ & 68.6 & 340 & $1.73 \cdot 10^{17}$ \\
\hline 2033 & 232 & $(58,88)$ & 113.4 & $(30,29,29)$ & 75.8 & 352 & $2.95 \cdot 10^{17}$ \\
\hline 2034 & 232 & $(58,88)$ & 113.4 & $(30,29,29)$ & 75.8 & 352 & $5.01 \cdot 10^{17}$ \\
\hline 2935 & 232 & $(58,88)$ & 113.4 & $(30,29,29)$ & 75.8 & 352 & $8.53 \cdot 10^{17}$ \\
\hline 2036 & 240 & $(60,91)$ & 125.3 & $(31,30,30)$ & 83.5 & 364 & $1.45 \cdot 10^{18}$ \\
\hline 2037 & 240 & $(60,91)$ & 125.3 & $(31,30,30)$ & 83.5 & 364 & $2.47 \cdot 10^{18}$ \\
\hline 2038 & 240 & $(60,91)$ & 125.3 & $(31,30,30)$ & 83.5 & 364 & $4.20 \cdot 10^{18}$ \\
\hline 2039 & 248 & $(62,94)$ & 138.0 & $(32,31,31)$ & 91.8 & 376 & $7.14 \cdot 10^{18}$ \\
\hline 2040 & 248 & $(62,94)$ & 138.0 & $(32,31,31)$ & 91.8 & 376 & $1.22 \cdot 10^{19}$ \\
\hline 2041 & 248 & $(62,94)$ & 138.0 & $(32,31,31)$ & 91.8 & 376 & $2.07 \cdot 10^{19}$ \\
\hline 2042 & 256 & $(64,97)$ & 151.6 & $(33,32,32)$ & 100.5 & 388 & $3.52 \cdot 10^{19}$ \\
\hline 3043 & 256 & $(64,97)$ & 151.6 & $(33,32,32)$ & 100.5 & 388 & $5.99 \cdot 10^{19}$ \\
\hline 2044 & 264 & $(66,100)$ & 166.0 & $(34,33,33)$ & 109.9 & 400 & $1.02 \cdot 10^{20}$ \\
\hline 2045 & 264 & $(66,100)$ & 166.0 & $(34,33,33)$ & 109.9 & 400 & $1.73 \cdot 10^{20}$ \\
\hline 2046 & 264 & $(66,100)$ & 166.0 & $(34,33,33)$ & 109.9 & 400 & $2.95 \cdot 10^{20}$ \\
\hline 2047 & 272 & $(68,103)$ & 181.3 & $(35,34,34)$ & 119.7 & 412 & $5.02 \cdot 10^{20}$ \\
\hline 2048 & 272 & $(68,103)$ & 181.3 & $(35,34,34)$ & 119.7 & 412 & $8.53 \cdot 10^{20}$ \\
\hline 2049 & 272 & $(68,103)$ & 181.3 & $(35,34,34)$ & 119.7 & 412 & $1.45 \cdot 10^{21}$ \\
\hline 2050 & 280 & $(70,106)$ & 197.5 & $(36,35,35)$ & 130.1 & 424 & $2.47 \cdot 10^{21}$ \\
\hline
\end{tabular}

Table 3. Proposed Parameters for Rainbow over GF(16) 


\begin{tabular}{|l|c|c|c|c|}
\hline years & $\begin{array}{c}\text { MinRank } \\
v_{1} \geq\end{array}$ & $\begin{array}{c}\text { HighRank } \\
o_{u} \geq\end{array}$ & $\begin{array}{c}\text { UOV-Attack } \\
n-2 o_{u} \geq\end{array}$ & $\begin{array}{c}\text { HR+UOV } \\
n \geq\end{array}$ \\
\hline $2010-2013$ & 16 & 17 & 18 & 52 \\
$2014-2016$ & 17 & 18 & 19 & 55 \\
$2017-2020$ & 18 & 19 & 20 & 58 \\
$2021-2023$ & 19 & 20 & 21 & 61 \\
$2024-2027$ & 20 & 21 & 22 & 64 \\
$2028-2030$ & 21 & 22 & 23 & 67 \\
$2031-2034$ & 22 & 23 & 24 & 70 \\
$2035-2037$ & 23 & 24 & 25 & 73 \\
$2038-2041$ & 24 & 25 & 26 & 76 \\
$2042-2044$ & 25 & 26 & 27 & 79 \\
$2045-2047$ & 26 & 27 & 28 & 82 \\
$2048-2051$ & 27 & 28 & 29 & 85 \\
\hline
\end{tabular}

Table 4. Parameter restrictions for Rainbow over GF(31) according to Proposition 1

further guessing at 1, 2, 3 or 4 additional variables we created overdetermined systems to see whether this reduces the time needed to compute a Gröbner Basis. When doing so, one has to run the algorithm several times to find a solution of the original system.

As table 5 shows, for more than 12 equations we get the best results when guessing at two

\begin{tabular}{|l|c|c|c|c|c|c|c|c|}
\hline \# equations & 11 & 12 & 13 & 14 & 15 & 16 & 17 & 18 \\
\hline no guessing & $7.8 \mathrm{~m}$ & $58.3 \mathrm{~m}$ & $7.7 \mathrm{~h}$ & $52.3 \mathrm{~h}$ & & & & \\
& $517 \mathrm{MB}$ & $1283 \mathrm{MB}$ & $7601 \mathrm{MB}$ & $53728 \mathrm{MB}$ & ooM & & & \\
\hline 1 guessed & $3.1 \mathrm{~m}$ & $18.9 \mathrm{~m}$ & $2.6 \mathrm{~h}$ & $15.8 \mathrm{~h}$ & $124.9 \mathrm{~h}$ & $846.5 \mathrm{~h}$ & & \\
& $13.3 \mathrm{MB}$ & $29.5 \mathrm{MB}$ & $82.4 \mathrm{MB}$ & $285 \mathrm{MB}$ & $979 \mathrm{MB}$ & $3872 \mathrm{MB}$ & & \\
\hline 2 guessed & $3.7 \mathrm{~m}$ & $25.7 \mathrm{~m}$ & $2.4 \mathrm{~h}$ & $14.4 \mathrm{~h}$ & $77.9 \mathrm{~h}$ & $428.8 \mathrm{~h}$ & $178.8 \mathrm{~d}$ & \\
& $8.7 \mathrm{MB}$ & $12.3 \mathrm{MB}$ & $17.3 \mathrm{MB}$ & $43.7 \mathrm{MB}$ & $108 \mathrm{MB}$ & $312 \mathrm{MB}$ & $1278 \mathrm{MB}$ & \\
\hline 3 guessed & & & $6.2 \mathrm{~h}$ & $38.2 \mathrm{~h}$ & $176.8 \mathrm{~h}$ & $726.5 \mathrm{~h}$ & $283.1 \mathrm{~d}$ & $1644.5 \mathrm{~d}$ \\
& & & $9.3 \mathrm{MB}$ & $15 \mathrm{MB}$ & $26 \mathrm{MB}$ & $53 \mathrm{MB}$ & $219 \mathrm{MB}$ & $587 \mathrm{MB}$ \\
\hline 4 guessed & & & & $70.8 \mathrm{~h}$ & $344.4 \mathrm{~h}$ & $1906.7 \mathrm{~h}$ & $556.4 \mathrm{~d}$ & $2994.5 \mathrm{~d}$ \\
& & & & $8.9 \mathrm{MB}$ & $10.8 \mathrm{MB}$ & $18 \mathrm{MB}$ & $43 \mathrm{MB}$ & $97 \mathrm{MB}$ \\
\hline
\end{tabular}

Table 5. Solving Rainbow systems over GF(31) by $F_{4}$ with guessing

variables. Furthermore, our extrapolation (see figure 3) shows that for $m \geq 25$ equations it is even better to guess at three variables. So, for the parameters currently used in multivariate schemes it is the optimal strategy to guess at three variables. Such we get

$$
R T_{F 4}(31, m)=2^{2.50 \cdot m-18.2} \sec (25 \leq m \leq 52)
$$

To have a secure Rainbow Scheme, this running time has to be greater or equal to our Security level, or

$$
m \geq \frac{\log _{2}(\text { Security level }(y))+18.2}{2.50}
$$

Note that in some cases the number $m$ given by formula (19) would lead to hash lengths which are not secure. In these cases the number of equations in our schemes is determined by the hash length.

RBS-attack To determine the number $n$ of variables needed in our Rainbow Schemes we carried out some experiments to estimate the running time of the Rainbow-Band-Separation (RBS) attack. 


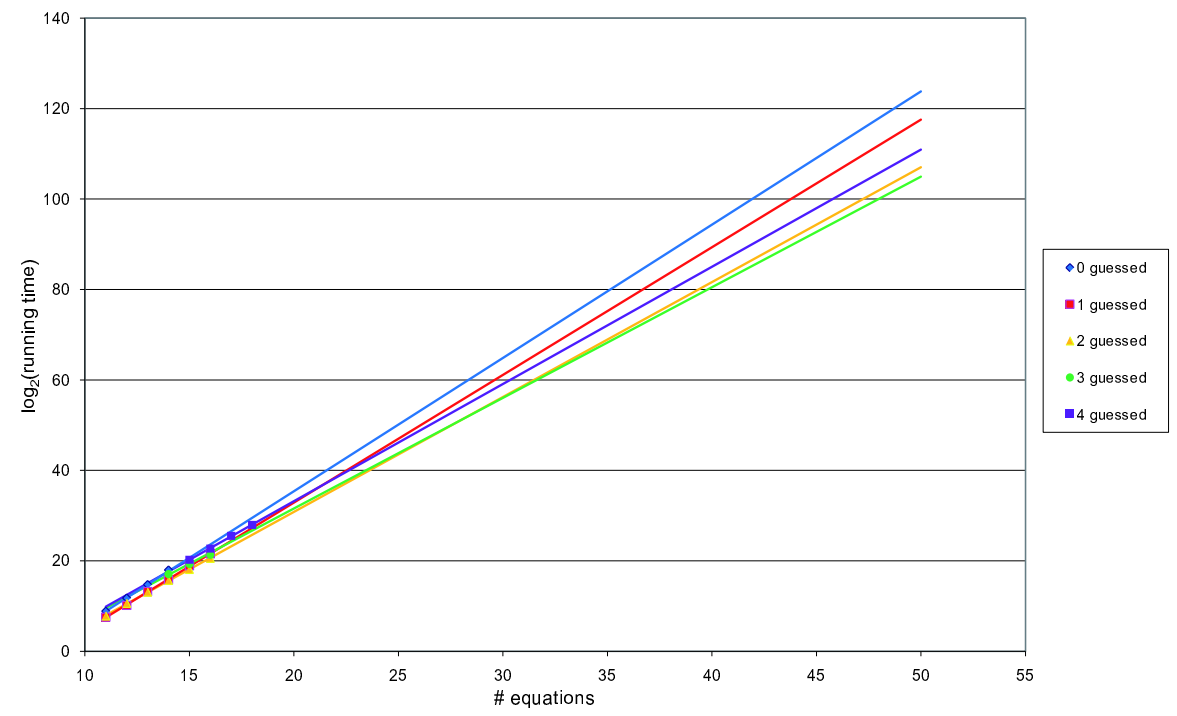

Fig. 3. Running time of the direct attack against Rainbow schemes over GF(31)

In the first step of this attack one has to solve an overdetermined system of $m^{\prime}=m+n-1$ equations in $n$ variables. The running time of the RBS attack is mainly given by the time needed to solve this system.

For different values of $m$ and $n$ we carried out experiments to find the time MAGMA needs to solve this initial system. Table 6 shows the results. As figure 4 shows, the running time of the RBS attack against a Rainbow Scheme with $m$ equations and $n=\frac{3}{2} \cdot(m-1)$ variables is almost the same as the running time of the direct attack against such a system (dotted line in the figure). Therefore, to create secure Rainbow Schemes over GF(31), we need

$$
n \geq \frac{3}{2} \cdot(m-1)
$$

Note that due to the UOV-attack we need often more variables than stated by this formula. So, in most cases the RBS-attack does not give a restriction to our parameter choice.

Data Conversion between $\boldsymbol{G F ( 3 1 )}$ and $\boldsymbol{G F}(2)^{\star}$ Since both hashvalues and signatures are usually given as bit strings, one needs to convert elements of $G F(2)^{\star}$ into elements of $G F(31)$ and vice versa. To store the keys, it is necessary to convert elements of $G F(31)$ into bitstrings, too. Like in [CC09] we use the following data conversion between $G F(31)$ and $G F(2)^{\star}$ :

- 3 elements of GF(31) fit into a 2-byte block

- an 8-byte block fits into 13 elements of GF(31) 


\begin{tabular}{|c||c|c|c|c|c|}
\hline \multirow{4}{*}{$n=2 \cdot(m-1)$} & $\mathrm{m}$ & 8 & 9 & 10 & 11 \\
\cline { 2 - 6 } & $\mathrm{n}$ & 14 & 16 & 18 & 20 \\
\cline { 2 - 6 } & & $34.1 \mathrm{~s}$ & $777 \mathrm{~s}$ & $9321 \mathrm{~s}$ & $153864 \mathrm{~s}$ \\
& & $30 \mathrm{MB}$ & $214 \mathrm{MB}$ & $765 \mathrm{MB}$ & $2890 \mathrm{MB}$ \\
\hline \hline \multirow{5}{*}{$n=\frac{5}{3} \cdot(m-1)$} & $\mathrm{m}$ & 7 & 10 & 13 & \\
\cline { 2 - 6 } & $\mathrm{n}$ & 10 & 15 & 20 & \\
\cline { 2 - 6 } & & $0.14 \mathrm{~s}$ & $50.4 \mathrm{~s}$ & $28921 \mathrm{~s}$ & \\
\hline \hline \multirow{3}{*}{$n=\frac{3}{2} \cdot(m-1)$} & & $0.7 \mathrm{MB}$ & $37 \mathrm{MB}$ & $2081 \mathrm{MB}$ & \\
\cline { 2 - 6 } & $\mathrm{m}$ & 9 & 11 & 13 & 15 \\
\cline { 2 - 6 } & $\mathrm{n}$ & 12 & 15 & 18 & 21 \\
\cline { 2 - 6 } & & $0.8 \mathrm{~s}$ & $40.5 \mathrm{~s}$ & $954 \mathrm{~s}$ & $56881 \mathrm{~s}$ \\
& & $9.2 \mathrm{MB}$ & $36 \mathrm{MB}$ & $231 \mathrm{MB}$ & $3291 \mathrm{MB}$ \\
\hline
\end{tabular}

Table 6. Running time of the RBS attack against Rainbow schemes over GF(31)

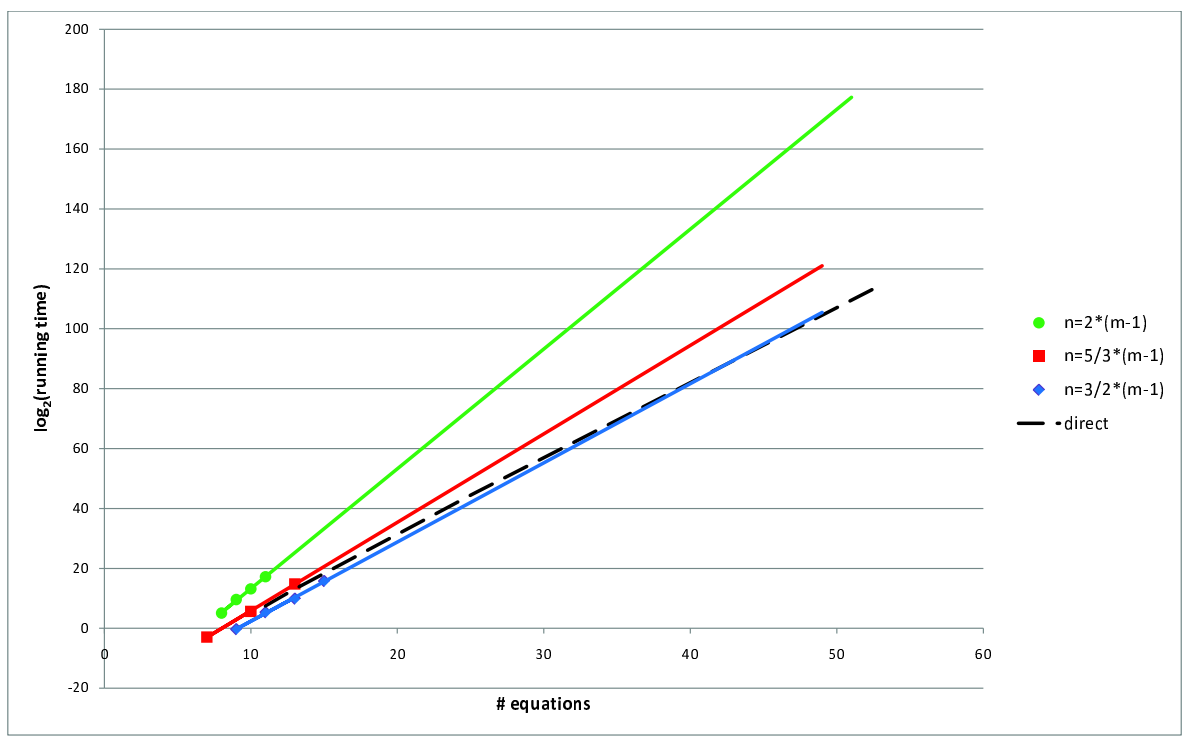

Fig. 4. Running time of the RBS attack against Rainbow schemes over GF(31) for different ratios of $m$ and $n$ 


\begin{tabular}{|c|c|c|c|c|c|c|c|c|c|}
\hline years & $\begin{array}{c}\text { hash } \\
\text { size (bit) }\end{array}$ & $(\mathrm{m}, \mathrm{n})$ & $\begin{array}{r}\text { public key siz } \\
\text { GF(31)-elements }\end{array}$ & $\mathrm{kB}$ & $\begin{array}{c}\text { example scheme } \\
\left(v_{1}, o_{1}, o_{2}\right)\end{array}$ & $\begin{array}{r}\text { private key siz } \\
\text { GF(31)-elements }\end{array}$ & $\mathrm{kB}$ & \begin{tabular}{|l|} 
signature \\
size (bit)
\end{tabular} & IMY \\
\hline 1982 & & & & & & & & & $5.00 \cdot 10^{5}$ \\
\hline 2010 & 160 & (33,52) & 47223 & 30.7 & $(19,16,17)$ & 34084 & 22.2 & 280 & $1.45 \cdot 10^{12}$ \\
\hline 2011 & 168 & $(35,52)$ & 50085 & 32.6 & $(17,18,17)$ & 34652 & 22.2 & 280 & $2.47 \cdot 10^{12}$ \\
\hline 2012 & 168 & $(35,52)$ & 50085 & 32.6 & $(17,18,17)$ & 34652 & 22.6 & 280 & $4.19 \cdot 10^{12}$ \\
\hline 2013 & 168 & $(35,55)$ & 55860 & 36.4 & $(20,18,17)$ & 39833 & 26.0 & 296 & $7.14 \cdot 10^{12}$ \\
\hline 2014 & 176 & $(36,55)$ & 57456 & 37.4 & $(19,18,18)$ & 40322 & 26.3 & 296 & $1.21 \cdot 10^{13}$ \\
\hline 2015 & 176 & $(36,55)$ & 57456 & 37.4 & $(19,18,18)$ & 40322 & 26.3 & 296 & $2.07 \cdot 10^{13}$ \\
\hline 2016 & 184 & $(38,58)$ & 67260 & 43.8 & $(20,19,19)$ & 46498 & 30.5 & 312 & $3.51 \cdot 10^{13}$ \\
\hline 2017 & 184 & $(38,58)$ & 67260 & 43.8 & $(20,19,19)$ & 46498 & 30.5 & 312 & $5.98 \cdot 10^{13}$ \\
\hline 2018 & 184 & $(38,58)$ & 67260 & 43.8 & $(20,19,19)$ & 46498 & 30.5 & 312 & $1.02 \cdot 10^{14}$ \\
\hline 2019 & 192 & $(39,58)$ & 69030 & 44.9 & $(19,20,19)$ & 47202 & 30.7 & 312 & $1.73 \cdot 10^{14}$ \\
\hline 2020 & 192 & $(39,58)$ & 69030 & 44.9 & $(19,20,19)$ & 47202 & 30.7 & 312 & $2.94 \cdot 10^{14}$ \\
\hline 2021 & 192 & $\mid(39,61)$ & 76167 & 44.5 & $(22,19,20)$ & 53749 & 35.0 & 328 & $5.01 \cdot 10^{14}$ \\
\hline 2022 & 200 & $(41,61)$ & 80073 & 45.8 & $(20,21,20)$ & 54476 & 35.5 & 328 & $8.52 \cdot 10^{14}$ \\
\hline 2023 & 200 & $(41,61)$ & 80073 & 45.8 & $(20,21,20)$ & 54476 & 35.5 & 328 & $1.45 \cdot 10^{15}$ \\
\hline 2024 & 200 & $(41,64)$ & 87945 & 51.7 & $(23,20,21)$ & 61676 & 40.2 & 344 & $2.47 \cdot 10^{15}$ \\
\hline 2025 & 208 & $(43,64)$ & 92235 & 51.7 & $(21,22,21)$ & 62460 & 40.7 & 344 & $4.20 \cdot 10^{15}$ \\
\hline 2026 & 208 & $(43,64)$ & 92235 & 53.1 & $(21,22,21)$ & 62460 & 40.7 & 344 & $7.14 \cdot 10^{15}$ \\
\hline 2027 & 208 & $(43,64)$ & 92235 & 54.5 & $(21,22,21)$ & 62460 & 40.7 & 344 & $1.21 \cdot 10^{16}$ \\
\hline 2028 & 216 & $(44,67)$ & 103224 & 59.6 & $(23,22,22)$ & 70798 & 46.1 & 360 & $2.07 \cdot 10^{16}$ \\
\hline 2029 & 216 & $(44,67)$ & 103224 & 61.1 & $(23,22,22)$ & 70798 & 46.1 & 360 & $3.52 \cdot 10^{16}$ \\
\hline 2030 & 224 & $(46,67)$ & 103224 & 61.1 & $(21,24,22)$ & 71508 & 46.6 & 360 & $5.98 \cdot 10^{16}$ \\
\hline 2031 & 224 & $(46,70)$ & 117576 & 68.2 & $(24,23,23)$ & 80272 & 52.3 & 376 & $1.02 \cdot 10^{17}$ \\
\hline 2032 & 224 & $(46,70)$ & 117576 & 68.2 & $(24,23,23)$ & 80272 & 52.3 & 376 & $1.73 \cdot 10^{17}$ \\
\hline 2033 & 232 & $(48,70)$ & 122688 & 69.9 & $(22,25,23)$ & 81037 & 52.8 & 376 & $2.95 \cdot 10^{17}$ \\
\hline 2034 & 232 & $(48,70)$ & 122688 & 71.6 & $(22,25,23)$ & 81037 & 52.8 & 376 & $5.01 \cdot 10^{17}$ \\
\hline 2935 & 232 & $(48,73)$ & 133200 & 77.7 & $(25,24,24)$ & 90554 & 59.0 & 392 & $8.53 \cdot 10^{17}$ \\
\hline 2036 & 240 & $(49,73)$ & 135975 & 79.5 & $(24,25,24)$ & 91002 & 59.2 & 392 & $1.45 \cdot 10^{18}$ \\
\hline 2037 & 240 & $(49,73)$ & 135975 & 79.5 & $(24,25,24)$ & 91002 & 59.2 & 392 & $2.47 \cdot 10^{18}$ \\
\hline 2038 & 240 & $(49,76)$ & 147147 & 95.8 & $(27,24,25)$ & 101124 & 65.8 & 408 & $4.20 \cdot 10^{18}$ \\
\hline 2039 & 248 & $(51,76)$ & 153153 & 88.0 & $(25,26,25)$ & 102156 & 66.5 & 408 & $7.14 \cdot 10^{18}$ \\
\hline 2040 & 248 & $(51,76)$ & 153153 & 89.9 & $(25,26,25)$ & 102156 & 66.5 & 408 & $1.22 \cdot 10^{19}$ \\
\hline 2041 & 248 & $(51,76)$ & 153153 & 91.9 & $(25,26,25)$ & 102156 & 66.5 & 408 & $2.07 \cdot 10^{19}$ \\
\hline 2042 & 256 & $(52,79)$ & 168480 & 99.1 & $(27,26,26)$ & 113674 & 74.0 & 424 & $3.52 \cdot 10^{19}$ \\
\hline 3043 & 256 & $(52,79)$ & 168480 & 101.3 & $(27,26,26)$ & 113674 & 74.0 & 424 & $5.99 \cdot 10^{19}$ \\
\hline 2044 & 264 & $(54,79)$ & 174960 & 101.3 & $(25,28,26)$ & 114616 & 74.6 & 424 & $1.02 \cdot 10^{20}$ \\
\hline 2045 & 264 & $(54,82)$ & 188244 & 111.2 & $(28,27,27)$ & 126578 & 82.4 & 440 & $1.73 \cdot 10^{20}$ \\
\hline 2046 & 264 & $(54,82)$ & 188244 & 113.9 & $(28,27,27)$ & 126578 & 82.4 & 440 & $2.95 \cdot 10^{20}$ \\
\hline 2047 & 272 & $(56,82)$ & 195216 & 127.1 & $(26,29,27)$ & 127583 & 83.1 & 440 & $5.02 \cdot 10^{20}$ \\
\hline 2048 & 272 & $(56,85)$ & 209496 & 136.4 & $(29,28,28)$ & 140422 & 91.4 & 456 & $8.53 \cdot 10^{20}$ \\
\hline 2049 & 272 & $(56,85)$ & 209496 & 136.4 & $(29,28,28)$ & 140422 & 91.4 & 456 & $1.45 \cdot 10^{21}$ \\
\hline 2050 & 280 & $(57,85)$ & 213237 & 138.8 & $(28,29,28)$ & 141000 & 91.8 & 456 & $2.47 \cdot 10^{21}$ \\
\hline
\end{tabular}

Table 7. Proposed Parameters for Rainbow over GF(31) 


\subsection{Rainbow Schemes over GF(256)}

In this Section we want to find the optimal parameters for the Rainbow Signature Scheme over $\mathrm{GF}(256)$.

Table 8 gives the parameter restrictions set by Rank and UOV attacks. To prevent attacks with the UOV-Reconciliation attack, one should also have $v_{1} \geq o_{u}$.

\begin{tabular}{|l|c|c|c|c|}
\hline years & $\begin{array}{c}\text { MinRank } \\
v_{1} \geq\end{array}$ & $\begin{array}{c}\text { HighRank } \\
o_{u} \geq\end{array}$ & $\begin{array}{c}\text { UOV-Attack } \\
n-2 o_{u} \geq\end{array}$ & $\begin{array}{c}\text { HR+UOV } \\
n \geq\end{array}$ \\
\hline 2010 & 9 & 10 & 11 & 31 \\
$2011-2015$ & 10 & 11 & 12 & 34 \\
$2016-2021$ & 11 & 12 & 13 & 37 \\
$2022-2027$ & 12 & 13 & 14 & 40 \\
$2028-2032$ & 13 & 14 & 15 & 43 \\
$2033-2038$ & 14 & 15 & 16 & 46 \\
$2039-2043$ & 15 & 16 & 17 & 49 \\
$2044-2049$ & 16 & 17 & 18 & 52 \\
$2050-2055$ & 17 & 18 & 19 & 55 \\
\hline
\end{tabular}

Table 8. Parameter restrictions for Rainbow over GF(256) according to Proposition 1

Direct attacks We carried out some experiments of solving Rainbow systems over $G F(256)$ with MAGMA's $F_{4}$ algorithm. Before we could apply the MAGMA function GroebnerBasis, we had to convert the underdetermined Rainbow systems into determined ones by guessing at some of the variables. By further guessing at 1,2,3 or 4 additional variables we created overdetermined systems to see whether this reduces the time needed to compute a Gröbner Basis. When doing so, one has to run the algorithm several times to find a solution of the original system. Table 9 shows the results of these experiments.

\begin{tabular}{|l|c|c|c|c|c|c|}
\hline \# equations & 11 & 12 & 13 & 14 & 15 & 16 \\
\hline \multirow{2}{*}{ no guessing } & $6.4 \mathrm{~m}$ & $0.8 \mathrm{~h}$ & $6.6 \mathrm{~h}$ & $47.2 \mathrm{~h}$ & - & - \\
& $342 \mathrm{MB}$ & $1236 \mathrm{MB}$ & $7426 \mathrm{MB}$ & $35182 \mathrm{MB}$ & ooM & \\
\hline \multirow{2}{*}{ guessing 1 variable } & $29 \mathrm{~m}$ & $2.8 \mathrm{~h}$ & $23 \mathrm{~h}$ & $134 \mathrm{~h}$ & $48 \mathrm{~d}$ & $257 \mathrm{~d}$ \\
& $11 \mathrm{MB}$ & $23 \mathrm{MB}$ & $76 \mathrm{MB}$ & $285 \mathrm{MB}$ & $997 \mathrm{MB}$ & $3953 \mathrm{MB}$ \\
\hline \multirow{2}{*}{ guessing 2 variables } & $264 \mathrm{~m}$ & $30 \mathrm{~h}$ & $170 \mathrm{~h}$ & $1214 \mathrm{~h}$ & $230 \mathrm{~d}$ & $1259 \mathrm{~d}$ \\
& $8.6 \mathrm{MB}$ & $10.7 \mathrm{MB}$ & $14.5 \mathrm{MB}$ & $42 \mathrm{MB}$ & $118 \mathrm{MB}$ & $335 \mathrm{MB}$ \\
\hline \multirow{2}{*}{ guessing 3 variables } & $5880 \mathrm{~m}$ & $715 \mathrm{~h}$ & $3830 \mathrm{~h}$ & $23597 \mathrm{~h}$ & $4449 \mathrm{~d}$ & $18443 \mathrm{~d}$ \\
& $8.3 \mathrm{MB}$ & $9.0 \mathrm{MB}$ & $11.2 \mathrm{MB}$ & $14.8 \mathrm{MB}$ & $24.8 \mathrm{MB}$ & $51.7 \mathrm{MB}$ \\
\hline \multirow{2}{*}{ guessing 4 variables } & $93807 \mathrm{~m}$ & $8126 \mathrm{~h}$ & $43465 \mathrm{~h}$ & $22652 \mathrm{~h}$ & $67129 \mathrm{~d}$ & $382986 \mathrm{~d}$ \\
& $7.9 \mathrm{MB}$ & $8.6 \mathrm{MB}$ & $10.6 \mathrm{MB}$ & $11.8 \mathrm{MB}$ & $12.9 \mathrm{MB}$ & $18.0 \mathrm{MB}$ \\
\hline
\end{tabular}

Table 9. Solving Rainbow systems over GF(256) with F4 with guessing

So, in our examples, we get the best results without guessing. But, as our extrapolation shows, for $m \geq 22$ equations it will be better to guess at one variable, and for $m \geq 29$ to guess at two variables before applying F4 (see figure 5). The time MAGMA needs for solving a determined 


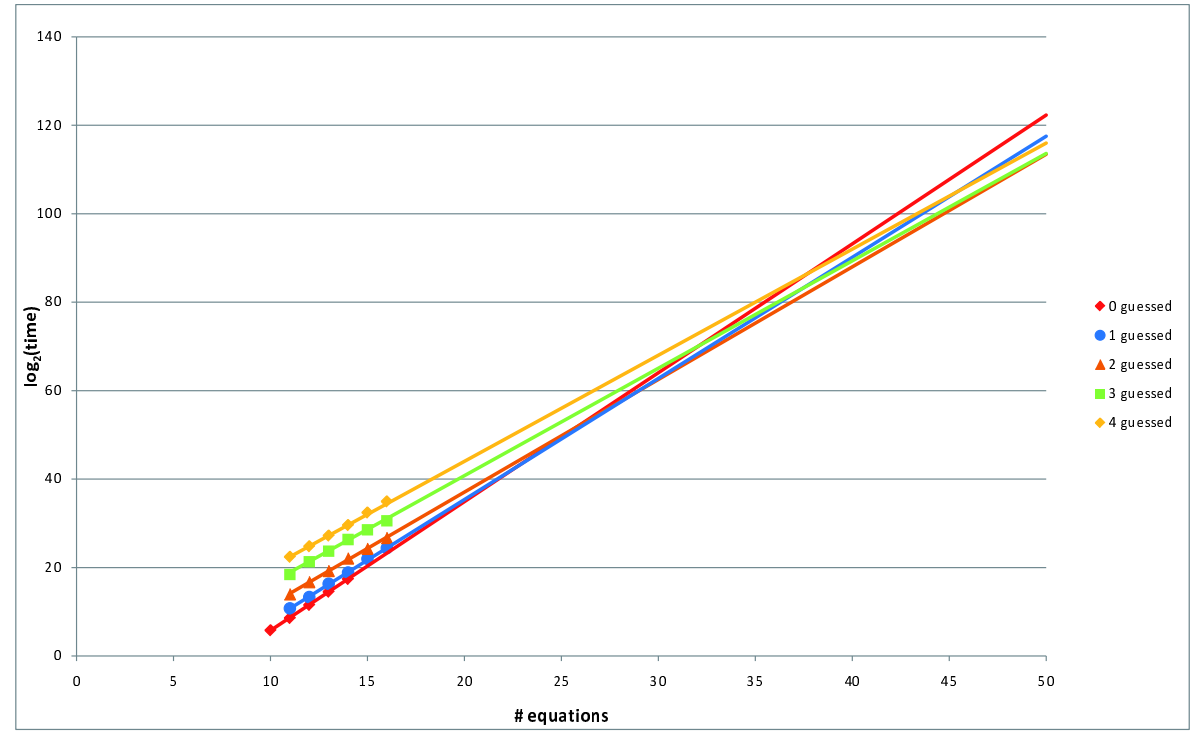

Fig. 5. Running time of the direct attack against Rainbow schemes over GF(256) with guessing

system with $m$ equations can then be estimated by the formula

$$
\begin{array}{ll}
\operatorname{RT}_{\mathrm{F} 4}\left(2^{8}, \mathrm{~m}\right)=2^{2.74 \cdot m-19.4} \mathrm{sec} & (22 \leq m \leq 28) \\
\operatorname{RT}_{\mathrm{F} 4}\left(2^{8}, m\right)=2^{2.55 \cdot m-13.9} \mathrm{sec} & (29 \leq m \leq 50)
\end{array}
$$

To have a secure Rainbow Scheme, this running time has to be greater or equal to our Security level, or

$$
m \geq \frac{\log _{2}(\operatorname{Security} \operatorname{level}(y))+13.9}{2.55}
$$

RBS-attack To determine the number $n$ of variables needed in our Rainbow Schemes we carried out some experiments to estimate the running time of the Rainbow-Band-Separation (RBS) attack. In the first step of this attack one has to solve an overdetermined system of $m^{\prime}=m+n-1$ equations in $n$ variables. The running time of the RBS attack is mainly given by the time needed to solve this system.

For different values of $m$ and $n$ we carried out experiments to find the time MAGMA needs to solve this initial system. Table 10 shows the results.

As Figure 6 shows, the running time of the RBS attack against a Rainbow Scheme over GF(256) with $m$ equations and $n=\frac{5}{3} \cdot(m-1)$ variables is almost the same as the running time of the direct attack against such a system (dotted line in the figure). Therefore, to create secure Rainbow Schemes over GF(256), we need

$$
n \geq \frac{5}{3} \cdot(m-1)
$$

Table 11 shows the proposed parameters for Rainbow over $G F(256)$. 


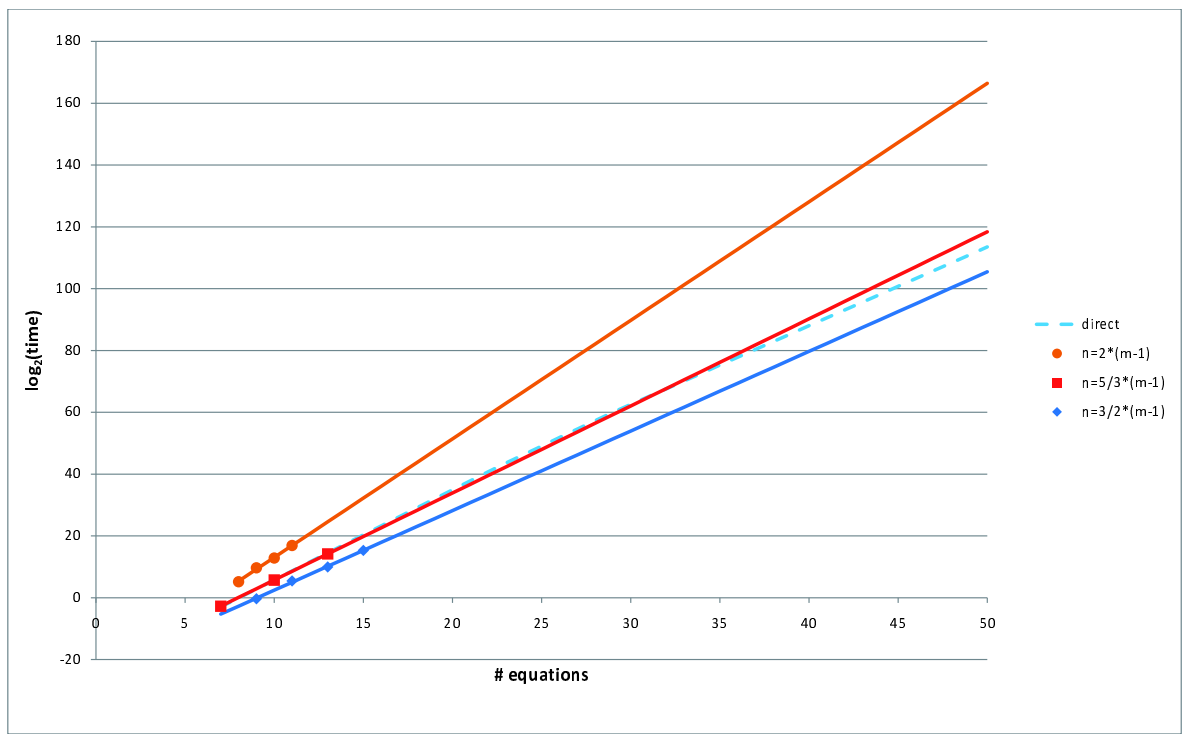

Fig. 6. Running time of the RBS attack against Rainbow schemes over GF(31) for different ratios of $m$ and $n$

\begin{tabular}{|c|c|c|c|c|c|}
\hline \multirow{4}{*}{$m=\frac{3}{2} \cdot r$} & \# equations & 21 & 24 & 27 & 30 \\
\hline & \# variables & 14 & 16 & 18 & 20 \\
\hline & & $36 \mathrm{~s}$ & $804 \mathrm{~s}$ & $7293 \mathrm{~s}$ & $120831 \mathrm{~s}$ \\
\hline & & $30 \mathrm{MB}$ & $214 \mathrm{MB}$ & $765 \mathrm{MB}$ & $2890 \mathrm{MB}$ \\
\hline \multirow{4}{*}{$m=\frac{8}{5} \cdot n$} & \# equations & 16 & 24 & 32 & \\
\hline & \begin{tabular}{|l}
$\#$ variables \\
\end{tabular} & 10 & 15 & 20 & \\
\hline & & $0.15 \mathrm{~s}$ & $52.5 \mathrm{~s}$ & $18263 \mathrm{~s}$ & \\
\hline & & $0.7 \mathrm{MB}$ & $37 \mathrm{MB}$ & $2081 \mathrm{MB}$ & \\
\hline \multirow{4}{*}{$m=\frac{5}{3} \cdot n$} & \# equations & 20 & 25 & 30 & 35 \\
\hline & \# variables & 12 & 15 & 18 & 21 \\
\hline & & $0.8 \mathrm{~s}$ & $42,7 \mathrm{~s}$ & $985 \mathrm{~s}$ & $40298 \mathrm{~s}$ \\
\hline & & $1.2 \mathrm{MB}$ & $36 \mathrm{MB}$ & $231 \mathrm{MB}$ & $3291 \mathrm{ME}$ \\
\hline
\end{tabular}

Table 10. Running time of the RBS attack against Rainbow over GF(256) 


\begin{tabular}{|c|c|c|c|c|c|c|c|}
\hline Year & $(m, n)$ & \begin{tabular}{|c|} 
public key \\
size $(\mathrm{kB})$ \\
\end{tabular} & $\begin{array}{c}\text { example scheme } \\
\left(v_{1}, o_{1}, o_{2}\right)\end{array}$ & $\begin{array}{c}\text { private key } \\
\text { size }(\mathrm{kB})\end{array}$ & $\begin{array}{c}\text { hash } \\
\text { size (bit) }\end{array}$ & \begin{tabular}{|c|} 
signature \\
size(bit) \\
\end{tabular} & IMY \\
\hline 1982 & & & & & & & $5.00 \cdot 10^{5}$ \\
\hline 2010 & $(26,43)$ & 25.7 & $(17,13,13)$ & 19.1 & 208 & 344 & $1.45 \cdot 10^{12}$ \\
\hline 2011 & $(27,45)$ & 29.2 & $(18,13,14)$ & 21.7 & 216 & 360 & $2.47 \cdot 10^{12}$ \\
\hline 2012 & $(27,45)$ & 29.2 & $(18,13,14)$ & 21.7 & 216 & 360 & $4.19 \cdot 10^{12}$ \\
\hline 2013 & $(28,46)$ & 31.6 & $(18,14,14)$ & 23.1 & 224 & 368 & $7.14 \cdot 10^{12}$ \\
\hline 2014 & $(29,47)$ & 34.1 & $(18,14,15)$ & 24.8 & 232 & 376 & $1.21 \cdot 10^{13}$ \\
\hline 2015 & $(29,47)$ & 34.1 & $(18,14,15)$ & 24.8 & 232 & 376 & $2.07 \cdot 10^{13}$ \\
\hline 2016 & $(30,49)$ & 38.3 & $(19,15,15)$ & 27.7 & 240 & 392 & $3.51 \cdot 10^{13}$ \\
\hline 2017 & $(30,51)$ & 41.3 & $(21,15,15)$ & 30.5 & 240 & 408 & $5.98 \cdot 10^{13}$ \\
\hline 2018 & $(31,52)$ & 44.4 & $(21,15,16)$ & 32.4 & 248 & 416 & $1.02 \cdot 10^{14}$ \\
\hline 2019 & $(31,52)$ & 44.4 & $(21,15,16)$ & 32.4 & 248 & 416 & $1.73 \cdot 10^{14}$ \\
\hline 2020 & $(32,53)$ & 47.3 & $(21,16,16)$ & 34.4 & 256 & 424 & $2.94 \cdot 10^{14}$ \\
\hline 2021 & $(33,54)$ & 50.8 & $(21,16,17)$ & 36.5 & 264 & 432 & $5.01 \cdot 10^{14}$ \\
\hline 2022 & $(33,55)$ & 52.7 & $(22,16,17)$ & 38.1 & 264 & 440 & $8.52 \cdot 10^{14}$ \\
\hline 2023 & $(34,57)$ & 58.2 & $(23,17,17)$ & 42.0 & 272 & 456 & $1.45 \cdot 10^{15}$ \\
\hline 2024 & $(34,58)$ & 60.2 & $(24,17,17)$ & 43.8 & 272 & 464 & $2.47 \cdot 10^{15}$ \\
\hline 2025 & $(35,59)$ & 64.1 & $(24,17,18)$ & 46.3 & 280 & 472 & $4.20 \cdot 10^{15}$ \\
\hline 2026 & $(35,59)$ & 64.1 & $(24,17,18)$ & 46.3 & 280 & 472 & $7.14 \cdot 10^{15}$ \\
\hline 2027 & $(36,60)$ & 68.1 & $(24,18,18)$ & 48.7 & 288 & 480 & $1.21 \cdot 10^{16}$ \\
\hline 2028 & $(37,61)$ & 72.3 & $(24,18,19)$ & 51.4 & 296 & 488 & $2.07 \cdot 10^{16}$ \\
\hline 2029 & $(37,63)$ & 77.0 & $(26,18,19)$ & 55.6 & 296 & 504 & $3.52 \cdot 10^{16}$ \\
\hline 2030 & $(38,65)$ & 84.0 & $(27,19,19)$ & 60.5 & 304 & 520 & $5.98 \cdot 10^{16}$ \\
\hline 2031 & $(38,65)$ & 84.0 & $(27,19,19)$ & 60.5 & 304 & 520 & $1.02 \cdot 10^{17}$ \\
\hline 2032 & $(39,66)$ & 88.8 & $(27,19,20)$ & 63.6 & 312 & 528 & $1.73 \cdot 10^{17}$ \\
\hline 2033 & $(39,66)$ & 88.8 & $(27,19,20)$ & 63.6 & 312 & 528 & $2.95 \cdot 10^{17}$ \\
\hline 2034 & $(40,68)$ & 96.7 & $(28,20,20)$ & 69.1 & 320 & 544 & $5.01 \cdot 10^{17}$ \\
\hline 2035 & $(40,69)$ & 99.4 & $(29,20,20)$ & 71.6 & 320 & 552 & $8.53 \cdot 10^{17}$ \\
\hline 2036 & $(41,72)$ & 110.7 & $(31,20,21)$ & 80.3 & 328 & 576 & $1.45 \cdot 10^{18}$ \\
\hline 2037 & $(42,73)$ & 116.6 & $(31,21,21)$ & 83.8 & 336 & 584 & $2.47 \cdot 10^{18}$ \\
\hline 2038 & $(42,73)$ & 116.6 & $(31,21,21)$ & 83.8 & 336 & 584 & $4.20 \cdot 10^{18}$ \\
\hline 2039 & $(43,74)$ & 122.6 & $(31,21,22)$ & 87.7 & 344 & 592 & $7.14 \cdot 10^{18}$ \\
\hline 2040 & $(43,74)$ & 122.6 & $(31,21,22)$ & 87.7 & 344 & 592 & $1.22 \cdot 10^{19}$ \\
\hline 2041 & $(44,76)$ & 132.1 & $(32,22,22)$ & 94.4 & 352 & 608 & $2.07 \cdot 10^{19}$ \\
\hline 2042 & $(44,78)$ & 139.0 & $(32,22,22)$ & 94.4 & 352 & 624 & $3.52 \cdot 10^{19}$ \\
\hline 2043 & $(45,79)$ & 145.8 & $(34,22,23)$ & 104.8 & 360 & 632 & $5.99 \cdot 10^{19}$ \\
\hline 2044 & $(46,80)$ & 152.8 & $(34,23,23)$ & 109.1 & 368 & 640 & $1.02 \cdot 10^{20}$ \\
\hline 2045 & $(46,80)$ & 152.8 & $(34,23,23)$ & 109.1 & 368 & 640 & $1.73 \cdot 10^{20}$ \\
\hline 2046 & $(47,81)$ & 159.9 & $(34,23,24)$ & 113.6 & 376 & 648 & $2.95 \cdot 10^{20}$ \\
\hline 2047 & $(47,82)$ & 163.8 & $(35,23,24)$ & 117.1 & 376 & 656 & $5.02 \cdot 10^{20}$ \\
\hline 2048 & $(48,84)$ & 175.4 & $(36,24,24)$ & 125.2 & 384 & 672 & $8.53 \cdot 10^{20}$ \\
\hline 2049 & $(48,85)$ & 179.6 & $(37,24,24)$ & 128.8 & 384 & 680 & $1.45 \cdot 10^{21}$ \\
\hline 2050 & $(49,85)$ & 183.3 & $(36,24,25)$ & 130.2 & 392 & 680 & $2.47 \cdot 10^{21}$ \\
\hline
\end{tabular}

Table 11. Proposed parameters for Rainbow over GF(256) 


\section{Summary}

In this section we summarize the results presented in the previous section. We compare Rainbow schemes over the three fields $G F(16), G F(31)$ and $G F(256)$ in terms of key sizes and signature lengths.

\subsection{Key Sizes}

Table 12 shows the public key sizes of Rainbow schemes over $G F(16), G F(31)$ and $G F(256)$.

\begin{tabular}{|c|c|c|c|}
\hline year & $\mathrm{GF}(16)$ & $\mathrm{GF}(31)$ & $\mathrm{GF}(256)$ \\
\hline 2010 & 38.1 & 30.7 & $\mathbf{2 5 . 7}$ \\
2020 & 65.0 & $\mathbf{4 4 . 9}$ & 47.5 \\
2030 & 102.3 & $\mathbf{7 2 . 3}$ & 84.0 \\
2040 & 138.0 & $\mathbf{9 9 . 7}$ & 122.6 \\
2050 & 197.5 & $\mathbf{1 3 8 . 8}$ & 183.3 \\
\hline
\end{tabular}

Table 12. Public key sizes of Rainbow over different fields (in $\mathrm{kB}$ )

At the moment, the key sizes are minimal for Rainbow Schemes over GF(256), but they increase much faster than the key sizes needed over GF(31). So, from the year 2018 on, the smallest keys are those of Rainbow schemes over GF(31).

\subsection{Signature Lengths}

Table 13 compares Rainbow schemes over $G F(16), G F(31)$ and $G F(256)$ in terms of the signature length.

\begin{tabular}{|c|c|c|c|}
\hline year & $\mathrm{GF}(16)$ & $\mathrm{GF}(31)$ & $\mathrm{GF}(256)$ \\
\hline 2010 & $\mathbf{2 4 4}$ & 280 & 344 \\
2020 & $\mathbf{2 9 2}$ & 312 & 424 \\
2030 & $\mathbf{3 4 0}$ & 360 & 520 \\
2040 & $\mathbf{3 7 6}$ & 408 & 592 \\
2050 & $\mathbf{4 2 4}$ & 456 & 680 \\
\hline
\end{tabular}

Table 13. Signature sizes for Rainbow over different fields (in bit)

As the table shows, one gets the shortest signatures when using Rainbow over GF(16). These signatures are about 20 to 30 bit shorter than the ones you get with $\operatorname{GF}(31)$. The signatures of Rainbow over $\mathrm{GF}(256)$ are much longer and this difference in length will increase over time. 


\section{Conclusion}

Although nobody can say, which cryptanalytic developments and developments in computing devices will take place in the next years, we hope that this paper will help people to choose 1) the field most suitable for their purpose and 2) appropriate parameters for the Rainbow signature scheme. The proposed parameter sets should give the reader an impression, which public key sizes are needed to achieve given levels of security.

\section{Acknowledgements}

We thank Jintai Ding, Bo-Yin Yang and Erik Dahmen for many helpful comments.

\section{References}

[BB08] Bernstein, D.J., Buchmann, J., Dahmen, E. (eds.): Post Quantum Cryptography. Springer, Heidelberg (2009)

[BC06] Bosma, W., Cannon, J., Playoust, C.: The Magma algebra system. I. The user language. J. Symbolic Comput., 24(3-4):235-265, 1997

[BG06] Billet, O., Gilbert, H.: Cryptanalysis of Rainbow. In DePrisco, R., Yung, M. (eds.) SCN 2006, LNCS vol. 4116, pp. 336-347. Springer, Heidelberg (2006)

[CC08] Chen, A.I.-T., Chen, C.-H. O., Chen, M.-S., Cheng, C.M., and Yang, B.-Y.: Practical-Sized Instances for Multivariate PKCs: Rainbow, TTS and $\ell$ IC- Derivatives. In: LNCS 5299 pp. 95-108, Springer Heidelberg (2008)

[CC09] Chen, A.I.-T., Chen, M.S., Chen T.R., Cheng, C.M., Ding, J., Kuo, E.L.H., Lee, F.Y.S., Yang B.-Y.: SSE-Implementation of Multivariate PKC's on Modern x86-CPUs. CHES 2009, pp. 33 -48

[CS94] Coppersmith, D., Stern, J., Vaudenay, S.: Attacks on the Birational Signature Scheme. In LNCS 773 pp. 435 to 443, Springer, Heidelberg (1994)

[DS05] Ding J., Schmidt D.: Rainbow, a new multivariate polynomial signature scheme. In Ioannidis, J., Keromytis, A.D., Yung, M. (eds.) ACNS 2005. LNCS vol. 3531, pp. 164-175 Springer, Heidelberg (2005)

[Di04] Ding, J.: A new variant of the Matsumoto-Imai cryptosystem through perturbation. In: Bao, F., Deng, R., Zhou, J. (eds.): PKC 2004, LNCS vol. 2947, pp. 266-281, Springer, Heidelberg (2004)

[DY08] Ding, J., Yang, B.-Y., Chen, C.-H. O., Chen, M.-S., and Cheng, C.M.: New Differential-Algebraic Attacks and Reparametrization of Rainbow. In: LNCS 5037, pp.242-257, Springer, Heidelberg (2005)

[DW07] Ding, J., Wolf, C., Yang, B.-Y.: $\ell$-invertible Cycles for Multivariate Quadratic Public Key Cryptography. In: Okamoto, T., Wang, X., (eds.): PKC 2007, LNCS, vol. 4450, pp. 266-281, Springer, Heidelberg (2007)

[DY07] Ding, J., Yang, B.-Y., Cheng, C.-M., Chen, O., and Dubois, V.: Breaking the symmetry: A way to resist the new Differential attacks. Available at http://www.eprint.iacr.org/2007/366.pdf

[Fa99] Faugere, J.C.: A new efficient algorithm for computing Groebner bases (F4). Journal of Pure and Applied Algebra, 139:61-88 (1999)

[Fa2] Faugere, J.C.: A new efficient algorithm for computing Groebner bases without reduction to zero (F5). In International Symposium on Symbolic and Algebraic Computation ISSAC 2002, pp. 75-83. ACM Press (2002)

[FP08] J.-C. Faugere, L. Perret: On the security of UOV. In: Proceedings of the First International Conference on Symbolic Computation and Cryptology, Beijing, 2008

[GC00] Goubin, L. and Courtois, N.T.: Cryptanalysis of the TTM cryptosystem. In Advances in Cryptology ASIACRYPT 2000, LNCS vol. 1976 , pp. 44-57. Tatsuaki Okamoto, ed., Springer (2000).

[GP09] G.-M. Greuel, G. Pfister and H. Schönemann: Singular 3.1.0 - A computer algebra system for polynomial computations, http://www.singular.uni-kl.de (2009)

[KP99] Kipnis, A., Patarin, L., Goubin, L.: Unbalanced Oil and Vinegar Schemes. In: Stern, J. (ed.) EUROCRYPT 1999. LNCS vol. 1592, pp. 206-222 Springer, Heidelberg (1999)

[KS98] Kipnis, A., Shamir, A.: Cryptanalysis of the Oil and Vinegar Signature scheme. In: Krawzyck, H. (ed.) CRYPTO 1998, LNCS vol. 1462, pp. 257-266 Springer, Heidelberg (1998)

[LV00] Lenstra, A.K., Verheul E.R.: Selecting Cryptographic Key Sizes. PKC 2000, pp. 446-465, www.keylength.com 
[MI88] Matsumoto, T., Imai, H.: Public Quadratic Polynomial-Tuples for efficient Signature-Verification and Message-Encryption. Advances in Cryptology - EUROCRYPT 1988, LNCS vol. 330, pp. 419-453, Springer, Heidelberg (1988)

[Pa96] Patarin, J.: Hidden Field equations (HFE) and Isomorphisms of Polynomials (IP). In: Proceedings of EUROCRYPT'96, pp. 38-48, Springer, Heidelberg (1996)

[Pa97] Patarin, J,: The oil and vinegar signature scheme, presented at the Dagstuhl Workshop on Cryptography (September 97)

[PG98] Patarin, J., Goubin, L., Courtois, N.: $C_{+}^{\star}$ and HM: Variations about two schemes of H. Matsumoto and T. Imai. In: Proceedings of ASIACRYPT'98, pp. 35-49, Springer, Heidelberg (1998)

[PC01] Patarin, J., Courtois, N., Goubin, L.: Flash, a fast multivariate signature algorithm. In C. Naccache, editor, Progress in cryptology, CT-RSA, LNCS vol. 2020, pp. 298-307. Springer, Heidelberg (2001)

[YC05] Yang, B.-Y., Chen J.-M.: Building secure tame like multivariate public-key cryptosystems: The new TTS. In: Boyd, C., Gonzales Nieto, J.M. (eds.) ACISP 2005. LNCS vol. 3574, pp. 518-531. Springer, Heidelberg (2005)

[YC07] Yang, B.-Y., Chen J.-M.: All in the XL family: Theory and practice. In LNCS 3506 pp. 67-86. Springer, Heidelberg (2007)

[Ya07] Yang, B.-Y., Chen, C.-H. O., Bernstein, D.J., and Chen, J.-M.: Analysis of QUAD. In: Biryukov, A. (ed.) FSE 2007, LNCS 4593 pp. 290-307. Springer, Heidelberg (2007) 